\title{
Olfactory blocking and odorant similarity in the honeybee
}

\author{
Fernando Guerrieri, ${ }^{1}$ Harald Lachnit, ${ }^{2}$ Bertram Gerber, ${ }^{3}$ and Martin Giurfa ${ }^{1,4}$ \\ ${ }^{1}$ Centre de Recherches sur la Cognition Animale, CNRS - Université Paul Sabatier Toulouse III - UMR 5169, F-31062 Toulouse \\ cedex 4, France; ${ }^{2}$ Fachbereich für Psychologie, Philipps-Universität Marburg, D-35032 Marburg, Germany; ${ }^{3}$ Institut für \\ Neurobiologie und Genetik, Biozentrum, Universität Würzburg, D-97074 Würzburg, Germany
}

\begin{abstract}
Blocking occurs when previous training with a stimulus $A$ reduces (blocks) subsequent learning about a stimulus $B$, when $A$ and $B$ are trained in compound. The question of whether blocking exists in olfactory conditioning of proboscis extension reflex (PER) in honeybees is under debate. The last published accounts on blocking in honeybees state that blocking occurs when odors A and B are similar (the "similarity hypothesis"). We have tested this hypothesis using four odors (1-octanol, 1-nonanol, eugenol, and limonene) chosen on the basis of their chemical and physiological similarity (experiment 1 ). We established a generalization matrix that measured perceptual similarity. Bees in the "block group" were first trained with an odor $A$ and, in the second phase, with the mixture $A B$. Bees in the "novel group" (control group) were first trained with an odor $N$ and, in the second phase, with the mixture $A B$. After conditioning, bees in both groups were tested for their response to $B$. We assayed all 24 possible combinations for the four odors standing for A, B, and N. We found blocking in four cases, augmentation in two cases, and no difference in 18 cases; odor similarity could not account for these results. We also repeated the experiments with those six odor combinations that gave rise to the similarity hypothesis (experiment 2: 1-hexanol, 1-octanol, geraniol) and found augmentation in one and no effect in five cases. Thus, blocking is not a consistent phenomenon, nor does it depend on odor similarity.
\end{abstract}

Understanding which conditions are necessary and sufficient for associative learning is one of the central issues of learning research. A major form of associative learning is classical conditioning (Pavlov 1927) in which animals learn to associate an originally neutral stimulus (conditioned stimulus [CS]) with a relevant stimulus (unconditioned stimulus [US]). The initial proposal was that contiguity of the CS and the US is sufficient to establish an association between them. This is, however, not the case as demonstrated by the phenomenon called blocking (Kamin 1968). In a blocking experiment, there are two acquisition phases. In the first phase (or preconditioning phase), subjects in the "block group" are exposed to a single CS A paired with an US. In the second phase (or compound-conditioning phase), subjects are exposed to a compound consisting of stimuli $\mathrm{A}$ and $\mathrm{B}$, paired with the US. Subjects in the "control group" are exposed in the first phase to novel stimulus $\mathrm{N}$ paired with the US, and to the compound $\mathrm{AB}$ paired with the US in the second phase. We call this group henceforth "novel group." Finally, in the test phase, subjects in both groups are presented with B alone. Blocking occurs if B elicits weaker responding in the block than in the control group despite the fact that B was identically paired with the US in both groups. It is then said that learning about stimulus A blocks subsequent learning about stimulus B when A and B are trained in compound.

Blocking had a profound influence on current theories of learning and behavior (see Rescorla and Wagner 1972; Mackintosh 1975; Pearce and Hall 1980; Miller and Matzel 1988) because it shows that the response elicited by a stimulus (B) is dependent of the associative status of another stimulus (A) that was present during training. It also shows that contiguity between a CS (B)

\section{${ }^{4}$ Corresponding author.}

E-mail giurfa@cict.fr; fax 33-561-55-6154.

Article and publication are at http://www.learnmem.org/cgi/doi/10.1101/ Im.79305. and the US is not always sufficient for learning. Furthermore, blocking constitutes an interesting phenomenon because it allows different explanatory levels for the absence of learning about B. One theory, proposed by Kamin (1969) and formalized in the Rescorla and Wagner model (1972), argues that blocking is caused by a lack of learning. According to this approach, in the second phase of training, there is lack of surprise about the occurrence of the reinforcer because stimulus A already predicts it. $B$ is therefore not learned. Another view of the blocking problem focuses on selective attentional processes, which make the animal focus on A and explicitly ignore $\mathrm{B}$ during $\mathrm{AB}$ training (Sutherland and Mackintosh 1971; Mackintosh 1975). This theory asserts that blocking is caused by a learned inattention to the blocked cue. Additionally, explanations of blocking at a perceptual level have been also proposed. It has been argued that perceptual similarity between A and B determines the occurrence of blocking (Smith and Cobey 1994; Linster and Smith 1997). If $\mathrm{A}$ and $\mathrm{B}$ are perceptually similar, first-phase training with A would engage a neural pathway for which B has later on to compete due to its similarity to A. In this case, neural coding of B will be impaired, thus leading to a blocking effect. The explanatory frame is put here not on learning but on the processing of sensory cues that are used as training stimuli.

Blocking has been studied in several vertebrates such as pigeons (Good and Macphail 1994), rats (Kamin 1968; Batsell 1996; Batsell and Batson 1999; Wiltrout et al. 2003), rabbits (Solomon 1977; Giftakis and Tait 1998; Allen et al. 2002), rhesus monkeys (Beauchamp et al. 1991; Waelti et al. 2001), and humans (Miller and Matute 1996; Arcediano et al 1997). In invertebrates, research on blocking has been conducted in the mollusks Limax (Sahley et al. 1981) and Hermissenda (Rogers and Matzel 1996), in the fruit fly Drosophila melanogaster, and in the honeybee Apis mellifera. In Drosophila, Brembs and Heisenberg (2001) used colors and patterns as CSs, and an aversive heat beam as US, and could not demonstrate the occurrence of blocking. In the hon- 
eybee, blocking was studied by using two main conditioning procedures: (1) conditioning of the approach flight toward a target in free-flying bees (von Frisch 1967), and (2) olfactory conditioning of the proboscis extension reflex (PER) in harnessed bees (Takeda 1961; Bitterman et al. 1983). The results of these experiments are contradictory.

By using free-flying bees, Funayama et al (1995) could not find blocking between colors and odors. They suggested that blocking should be observable with stimuli of the same modality but not of different modalities. Couvillon et al. (1997) tested and demonstrated this assumption by using compounds of two colors, of two odors, and of position (visual proximity to a visible landmark) and color. In all three cases, the response of bees was consistent with blocking. When the compound was intermodal (odor-position) however, no blocking was found (Couvillon et al. 1997). Later, Couvillon et al. (2001) found blocking between intermodal stimuli, but such an effect depended on stimulus directionality; i.e., odor blocked position but neither position nor color blocked odor. This difference was explained in terms of relative stimulus salience, with odor being more salient than color and landmark (Couvillon et al. 2001).

Intramodal blocking was extensively studied by using olfactory conditioning of PER. In olfactory PER conditioning, harnessed bees are trained to associate an odor with a reward of sucrose delivered to their antennae, which elicits PER (Takeda 1961; Bitterman et al. 1983). The association formed enables the odor to release PER in a following test. In this case, a controversy exists concerning the existence of blocking. While blocking was reported in some cases (Smith and Cobey 1994; Smith 1997a; Thorn and Smith 1997; Hosler and Smith 2000), its existence was not confirmed in other cases (Gerber and Ullrich 1999). The first report on olfactory blocking in bees (Smith and Cobey 1994) concluded that conditioning a first odorant blocks learning about a second odorant presented in a binary compound with the first one. This conclusion was refuted by Gerber and Ullrich (1999), who identified two procedural confounds in the design of Smith and Cobey (1994): a reduction of the intertrial interval from the first to the second phase of training, and an absence of balance of the odorants used. When these two confounding factors were controlled, blocking did not occur (Gerber and Ullrich 1999). Counterbalancing may not be so critical as subsequent works (Thorn and Smith 1997; Smith 1997a) did fully counterbalance the design and reported nevertheless blocking. Later, Hosler and Smith (2000) replied that blocking does indeed occur in the olfactory modality, but it is in fact restricted to perceptually similar odors (the "similarity hypothesis"; see above). However, some procedural aspects of the work of Hosler and Smith (2000) were unclear. First, they used three odorants (geraniol, 1-hexanol, 1-octanol), arranged in only six odor combinations. It seems risky to validate a hypothesis on the role of odorant similarity on the basis of this reduced number of cases, especially because there were only two cases in which $\mathrm{A}$ and $\mathrm{B}$ were similar (A, 1-hexanol; B, 1-octanol and vice versa). Furthermore, in one case in which A and B were not similar (A, geraniol; B, 1-hexanol), blocking was nevertheless found (see Fig. 4 in Hosler and Smith 2000). Second, the amount of individuals per group, block or novel, was 10 for each odor combination, a fact that could weaken the statistical analysis. Third, the investigators had a block group and a novel group (control) in which bees were first trained with an odorant $\mathrm{N}$, different from $\mathrm{A}$ and $\mathrm{B}$, and afterward with $\mathrm{AB}$. They stated that blocking does not occur whenever the response to $\mathrm{B}$ in the novel and the block group was similar. However, they did not discuss that in two of the three cases in which blocking was said not to occur, the response to B was indeed similar in both the novel and the block groups, but simply because in both cases bees did not respond to B (see Fig. 4B,C of
Hosler and Smith 2000). The odorant stimulation lasted 4 sec and the response measured reached barely $1 \mathrm{sec}$ in both cases, while response duration is normally twice the duration of the CS stimulation (see Fig. 4A,E of Hosler and Smith 2000). Thus, we think that under these circumstances, it is not legitimate to state that blocking did not occur.

We therefore readdressed the question of blocking in olfactory conditioning of PER in bees. Given that the last account on the debate about the existence of olfactory blocking in honeybees is the similarity hypothesis postulated by Linster and Smith (1997) and Hosler and Smith (2000), and the criticism formulated above regarding the experimental basis supporting this hypothesis, we decided to test the similarity hypothesis strictly following the procedure of Hosler and Smith (2000). To this end, we studied performance in 24 (and not six) odor combinations resulting from four odorants (experiment 1). Furthermore we repeated the experiments of Hosler and Smith (2000) by using the same odors and groups (experiment 2 ). In each group, the number of bees was 20 (instead of 10). Different from Hosler and Smith (2000) we did not measure PER duration but frequency of PER occurrence; the increased sample size can, at the very least, come up for any presumably reduced sensitivity of the percentage of PER (\% PER) measure; this is clearly shown by the fact that we can indeed find between-group differences with this measure.

A
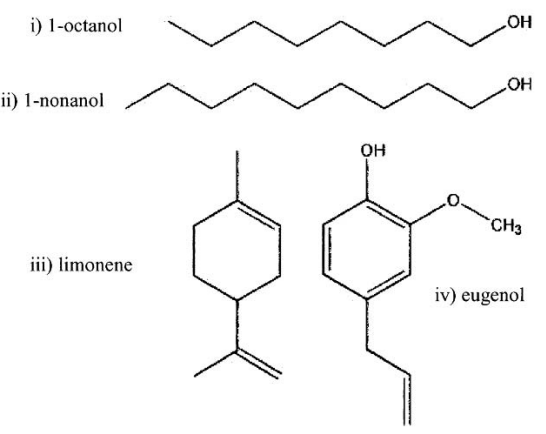

B

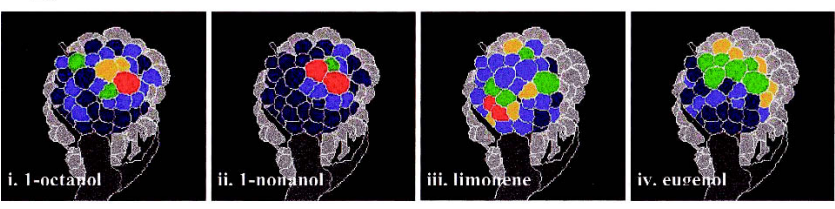

C

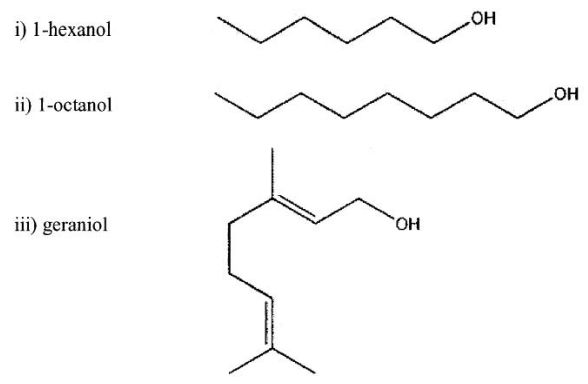

Figure 1. The four odors used in experiment 1: (1) 1-octanol, (2) 1-nonanol, (3) limonene, and (4) eugenol. (A) Molecular structures. (B) Glomerular activation patterns (neural olfactory coding) at the level of the antennal lobe, the primary olfactory neuropile of the bee brain (courtesy of G. Galizia) (for explanations about the optophysiological measurements of antennal lobe activity, see Galizia and Menzel 2000). (C) Molecular structures of the odors used in experiment 2 (same as in Hosler and Smith 2000). 


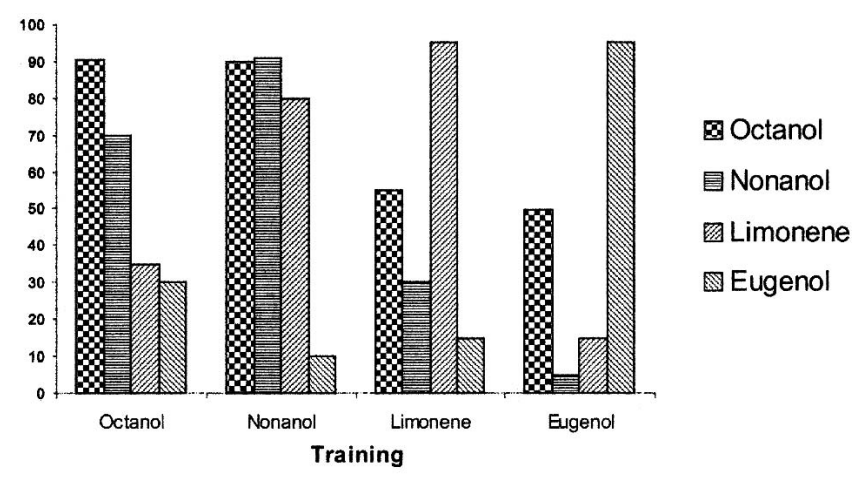

Figure 2. Experiment 1: Behavioral generalization matrix. The percentage of PER in the single extinction test is represented for the four odors tested as a function of the trained odor. In the four cases in which the odor tested corresponded to the odor trained (e.g., training with 1octanol, test of 1-octanol), the number of bees per column is 60 . In the other cases, the number of bees per column is 20 .

\section{Results}

We performed two main experiments in order to test the role of odor similarity in the occurrence of blocking in the honeybee. In the first one (experiment 1), we used odors chosen on the basis of their chemical and physiological similarity and defined a generalization matrix to quantify behavioral similarity. We then studied the occurrence of blocking depending on odor similarity, following the procedure of Hosler and Smith (2000). In the second one (experiment 2), we explicitly repeated Hosler and Smith's (2000) study.

\section{Experiment 1}

\section{Measure of similarity: Behavioral generalization matrix}

We first established a behavioral generalization matrix for the four odorants used in our experiments: 1-octanol, 1-nonanol, limonene, and eugenol (Fig. 1A).

Besides inferences on similarity based on their chemical structure (Fig. 1A) and on the neural activity patterns (Fig. 1B) they evoke in the olfactory neuropiles of the bee brain (Joerges et al. 1997; Galizia and Menzel 2000), the matrix allowed quantifying perceptual similarity and determining possible asymmetries between odors. Each of the four odors was conditioned in the course of six trials. Bees were then presented with a different odorant in a single extinction test. Different from the repeatedtesting procedure of Hosler and Smith (2000), a different group of fresh bees was used for each possible odor combination. In order to have the response to the same odorant, we considered for each of the four odors the response in the last acquisition trial. Figure 2 shows that the response level to the odors presented in the test varied depending on the odor used in the training. The highest response in the test was always to the odor used during the training (1,octanol: $\chi^{2}=82.29, d f=3, P<0.0001 ; 1$-nonanol: $\chi^{2}=$ 65.32, $d f=3, P<0.0001$; limonene: $\chi^{2}=98.71, d f=3, P<0.0001$; eugenol: $\left.\chi^{2}=118.52, d f=3, P<0.0001\right)$.

Taking into account the responses in the tests, we could establish different degrees of generalization between odors. In five of six cases (1-octanol and 1-nonanol; 1-octanol and limonene; 1-octanol and eugenol; 1-nonanol and eugenol; limonene and eugenol), generalization was symmetric; i.e., the response to $B$ after training of A did not differ significantly from the response to A after training to B ( $P>0.05$ in all five cases); therefore, data for both directions were pooled for subsequent analyses. Only in the case of 1-nonanol and limonene, generalization was asymmetric: Bees responded significantly more from 1-nonanol to li- monene than from limonene to 1-nonanol $\left(2 \times 2 \chi^{2}=4.5455\right.$, $P<0.04)$.

In order to rank generalization levels between odors, we compared them by means of $\chi^{2}$ analyses (Fig. 3A). This allowed to establish the following rank order of similarity based on generalization levels: (1) high-between 1-octanol and 1-nonanol, and from 1-nonanol to limonene (rank 3 for $\mathrm{O}-\mathrm{N}$ and $\mathrm{N} \rightarrow \mathrm{L}$ ); (2) intermediate-between 1-octanol and limonene, between 1-octanol and eugenol, and from 1-nonanol to limonene (rank 2 for $\mathrm{O}-\mathrm{L}, \mathrm{O}-\mathrm{E}$, and $\mathrm{L} \rightarrow \mathrm{N}$ ); and (3) low-between limonene and eugenol, and between 1-nonanol and eugenol (rank 1 for L-E and $\mathrm{N}-\mathrm{E}$ ). Similarities between odors are depicted in Figure 3B in which the four triads used in the blocking experiments are presented.

\section{Blocking: Global performance}

The design of the blocking experiments followed that of Hosler and Smith (2000). For each odor combination, we studied two groups during two conditioning phases (Table 1).

In the first training phase (preconditioning phase), the block group was preconditioned to odorant $\mathrm{A}$, while the novel group was preconditioned to a different odorant $\mathrm{N}$. In the second training phase (compound-conditioning phase), both groups were conditioned with a compound of two odorants $\mathrm{AB}$. After the last conditioning trial, both groups were tested with $\mathrm{B}$ in a single extinction trial. The response to B in both groups was then compared. If blocking occurred, the response to B should be lower in the block group than in the novel group despite the fact that both groups experienced $\mathrm{B}$ in an identical manner. Odorants were used equally often and were counterbalanced across days, as N, A, and B. Twenty-four independent experiments (i.e., 24 block and 24 novel groups) were performed ( 20 bees per group, 960 bees in

A

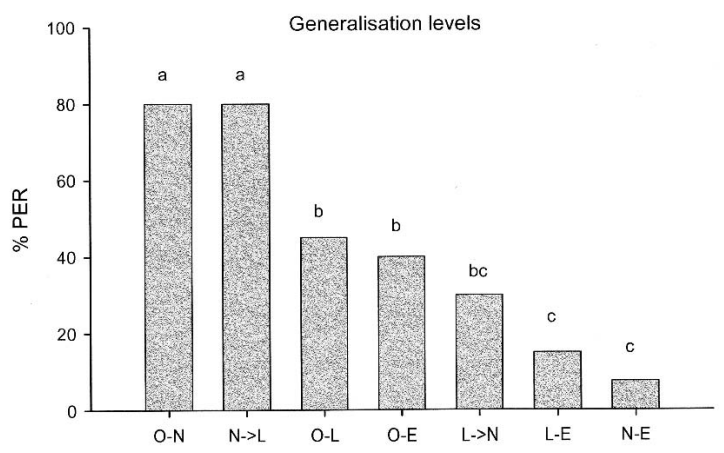

B
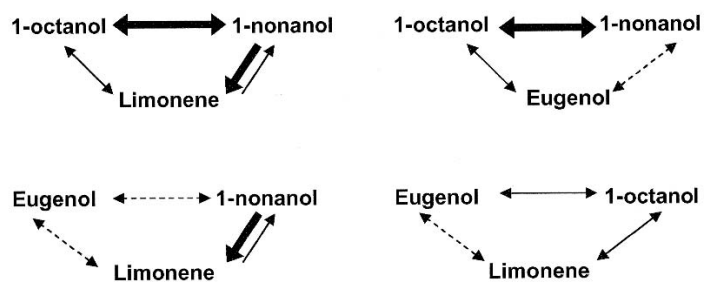

Figure 3. Experiment $1(A)$ Generalization between odor pairs. Bars which do not differ at 0.05 are indicated with same letter: 0,1 -octanol; $\mathrm{N}$, 1-nonanol; L, limonene; and $\mathrm{E}$, eugenol. $(B)$ The four triads of odors used in the blocking experiments and the generalization levels between odors within each triad. A double-headed arrow indicates symmetric generalization. A single-headed arrow indicates asymmetric generalization. Thick line indicates higher generalization; thin line, intermediate generalization; and dashed line, low generalization. 
Table 1. Experimental design of the blocking experiment

\begin{tabular}{lccc}
\hline Group & First phase & $\begin{array}{c}\text { Compound } \\
\text { conditioning }\end{array}$ & Test \\
\hline Block & $\mathrm{A}+$ & $\mathrm{AB}+$ & $\mathrm{B} ?$ \\
Novel & $\mathrm{N}+$ & $\mathrm{AB}+$ & $\mathrm{B} ?$ \\
\hline
\end{tabular}

After the method of Hosler and Smith (2000).

total). Differences in odor similarity between odorants allowed testing the effect of olfactory similarity on blocking.

In a first overall analysis that focused exclusively on the pooled performance in the test, we found that there was a blocking-like effect because the response given by the bees trained in the novel groups, pooled all together $(M=0.62 ; n=480$ bees $)$, was significantly higher $\left(F_{(1,912)}=5.21 ; P<0.03 ; \eta^{2}=0.006\right)$ than the response given by the bees of the block groups (Fig. 4, right panel) pooled altogether $(M=0.55 ; n=480$ bees). This blocking effect was remarkably weak (as $\eta^{2}<0.01$ is considered small) and could be related to the large sample size (960 individuals) used in our experiments.

We also found that there was a significant difference between the different odors combinations used $\left(F_{(23,912)}=7.17\right.$; $P<0.0001 ; \eta^{2}=0.153 ; \eta^{2}>0.138$ is considered strong) and that the interaction between the two factors was also significant $\left(F_{(23,912)}=2.44 ; P<0.0002 ; \eta^{2}=0.058 ; \eta^{2}\right.$ of $\sim 0.059$ is considered intermediate). Thus, the occurrence of blocking depends on the odor combination used. Hosler and Smith (2000) employed a similar argumentation and put the accent on odor similarity as the feature defining the occurrence of blocking.

In a second overall analysis, we compared the pooled performance of the bees of all block groups in the last trial of the preconditioning phase and in the first trial of the compoundconditioning phase. Due to explicit preconditioning in the first phase, odor A should have a high associative strength at the beginning of the compound training phase. Odor $\mathrm{B}$, on the other hand, should have no associative strength. If bees are able to identify $\mathrm{A}$ in the compound $\mathrm{AB}$, then, ideally, their first anticipatory response to $\mathrm{AB}+$ should not differ from the anticipatory response to the last presentation of $\mathrm{A}+$ in the preconditioning phase. Figure 4 (left and middle panels) shows that the level of responding in the first compound-conditioning trial $(M=0.59$, $n=480$ bees) was significantly lower than that in the last trial of the first training phase $\left(M=0.82, n=480\right.$ bees; $F_{(1,456)}=161.29$; $P<0.0001, \eta^{2}=0.261$ ). This result suggests that bees did not generalize fully between $\mathrm{A}$ and $\mathrm{AB}$. The main effect "odor combination" $\left(F_{(23,456)}=3.73 ; P<0.0001 ; \eta^{2}=0.158\right)$ and the interaction
$\left(F_{(23,456)}=4.91 ; P<0.0001 ; \eta^{2}=0.198\right)$ were also significant. This underlines again the necessity of an odor-combination based analysis. In the next section, we therefore considered the specific effect of each odor combination, and in particular, the effect of odor similarity.

\section{Blocking and odor similarity}

We asked whether blocking increases with increasing odorant similarity. Based on our behavioral generalization matrix, we regrouped the 24 odor combinations in three classes of similarity (rank 1 to 3 [see above], implying ascending similarity of odors A and B) and presented the performance of both the novel and the block groups in the three phases of the experiment: preconditioning, conditioning, and test (Fig. 5). If the similarity hypothesis of Linster and Smith (1997) and Hosler and Smith (2000) is correct, responding to $\mathrm{B}$ in the test phase in the block groups should decrease with increasing similarity between A and B because the more similar the odors are, the more blocking should occur.

The analyses showed comparable significant linear trends both for the block and the novel group (block: $F_{(1,477)}=8.80$, $P=0.003$; novel: $\left.F_{(1,477)}=7.35, P=0.007\right)$. Responding to $\mathrm{B}$ in the block groups in the test was highest when similarity to A was high $(M=0.66, n=120)$, intermediate when similarity was intermediate $(M=0.55, n=200)$, and lowest when similarity was low $(M=0.48, n=160)$. Responding to $B$ in the novel group followed a similar trend: It was highest when similarity to A was high ( $M=0.71, n=120)$, intermediate when similarity was intermediate $(M=0.62, n=200)$, and lowest when similarity was low $(M=0.55, n=160)$. Thus, in contradiction to the similarity hypothesis no decrease in the response to $\mathrm{B}$ in the block groups was found in the test phase as depending on the similarity between A and $\mathrm{B}$. The direction of the observed trend, however, may well be explained by generalization from the compound $\mathrm{AB}$ to $\mathrm{B}$, as an equivalent trend was observed for the novel group. Moreover, the difference between the block and the novel groups (the blocking effect) did not vary systematically as depending on similarity of A to B (high similarity: 0.05 [0.71-0.66]; intermediate similarity: 0.07 [0.62-0.55]; low similarity: 0.07 [0.55-0.48]).

\section{Blocking: Performance and odor combination}

We next looked for blocking within each of the 24 odor combinations. We looked for differences between the groups block and novel in their response to $\mathrm{B}$ in the test phase. When multiple comparisons are done, a threshold correction that takes into account the number of comparisons is usually applied for determining the significance level. As we had 24 odor combinations,
First training phase

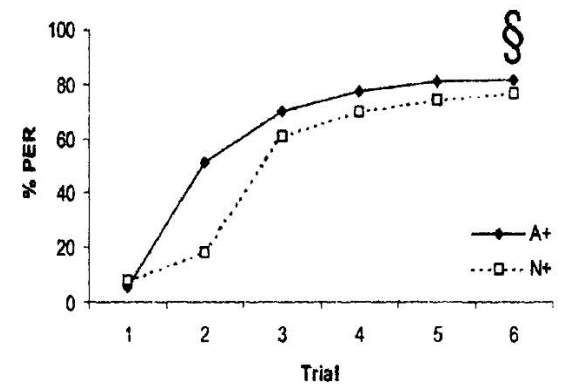

Compound conditioning

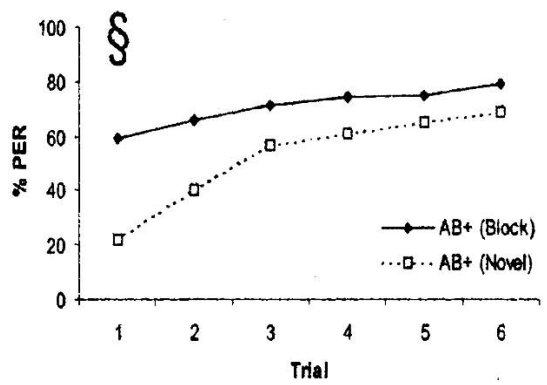

Test

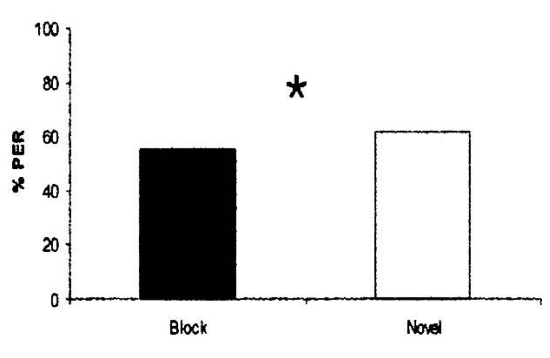

Figure 4. Experiment 1. Global analysis of blocking (pooled data for training and test). (Left) Percentage of PER in the six conditioning trials of the first training phase (preconditioning with a single odor, A or N). (Middle) Percentage of PER in the six conditioning trials of the second training phase (compound conditioning with $\mathrm{AB}$ ). §Significant difference between sixth preconditioning trial and the first compound-conditioning trial. (Right) Percentage of PER in the extinction test. Response to B was significantly higher in the novel than in the block group. 

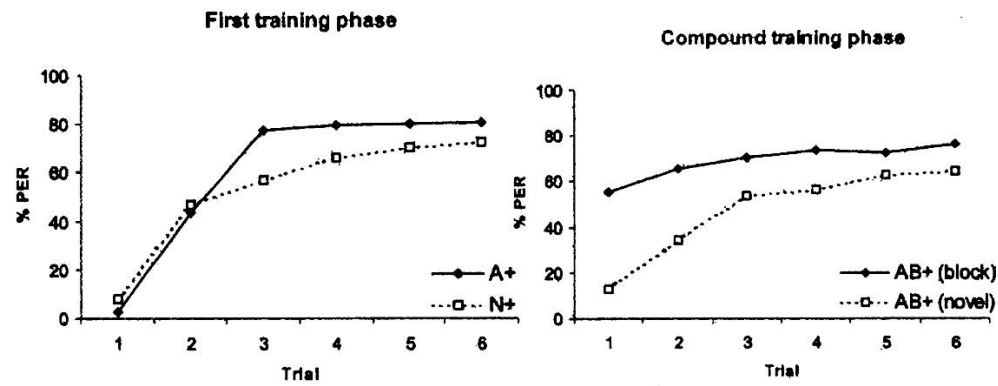

\section{Intermediate similarity}
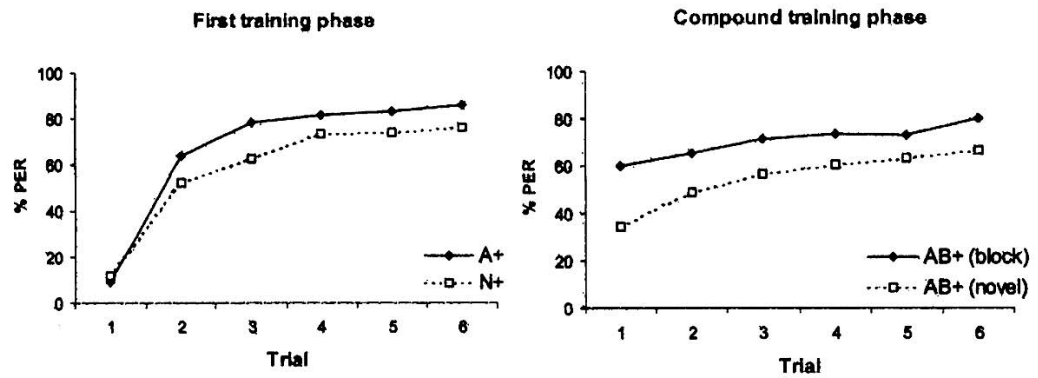

\section{High similarity}
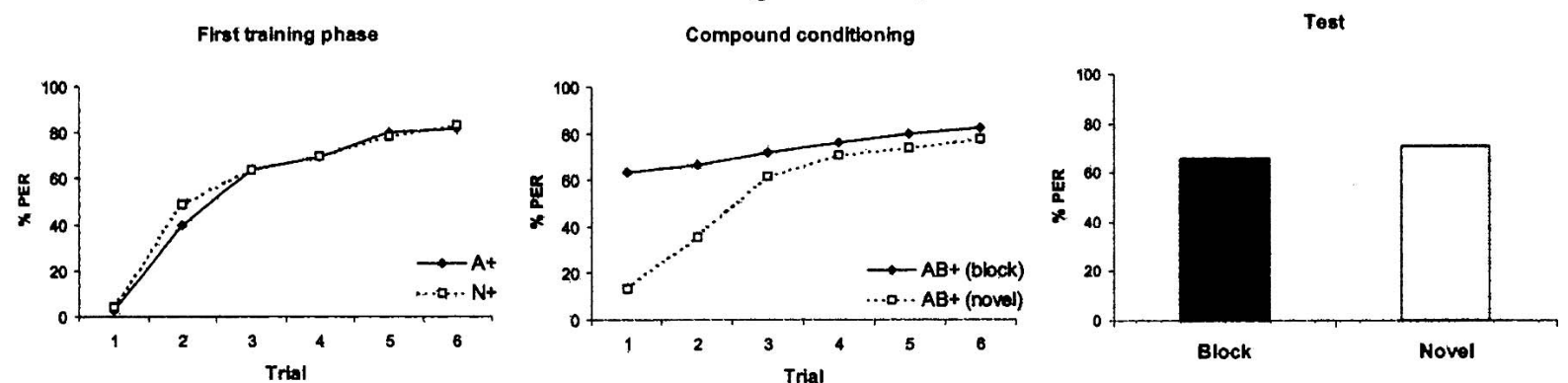

Figure 5. Experiment 1: Blocking and odor similarity. Odor combinations were pooled according to their similarity (high, intermediate, and low) as established through the generalization matrix. (Left) Percentage of PER in the six conditioning trials of the first training phase (preconditioning with a single odor, A or N). (Middle) Percentage of PER in the six conditioning trials of the second training phase (compound conditioning with AB). (Right) Percentage of PER in the extinction test.

applying this correction yields an $\alpha$ of $0.0021(0.05 / 24)$. If this $\alpha$ value is taken, no significant difference is observed in any of the 24 test comparisons between block and novel groups. This result is consistent with our statement about the weakness of the blocking phenomenon (see above) and with previous reports questioning the existence of blocking (Gerber and Ullrich 1999). However, if we use a noncorrected $\alpha$ of 0.05 for our comparisons (as done by Hosler and Smith 2000), we find different trends depending on the odors considered.

\section{Blocking-like test performances ( $B_{\text {block }}<B_{\text {novel }}$ )}

We found four odor combinations out of the 24 consistent with the definition of blocking because the response to $\mathrm{B}$ in the test was significantly higher in the novel than in the block group (Table 2; mid column; $P=<0.05$ in all cases).

These were as follows: (1) $\mathrm{N}=$ limonene, $\mathrm{A}=$ eugenol, $\mathrm{B}=1$ octanol; (2) N = 1-nonanol, $\mathrm{A}=$ limonene, and $\mathrm{B}=$ 1-octanol; (3) $\mathrm{N}=$ eugenol, $\mathrm{A}=1$-octanol, and $\mathrm{B}=1$-nonanol; and (4) $\mathrm{N}=1$ nonanol, $\mathrm{A}=1$-octanol, and $\mathrm{B}=$ eugenol. Again, the argument of odor similarity as determinant of blocking must be refuted because in three out of the four cases, A and B were not similar. The odor pairs eugenol and 1-octanol, limonene and 1-octanol, as well as 1-octanol and eugenol have different molecular structures (Fig. 1A), induce different neural activation patterns (Fig. 1B), and result in intermediate levels of behavioral generalization (Fig. 3). Odors of only one out of the four pairs (1-octanol and nonanol) can be considered as similar. Even this result does not support the similarity argument as no blocking was found in the combination in which the identities of $\mathrm{A}$ and $\mathrm{B}$ were inverted ( $\mathrm{N}=$ eugenol, $\mathrm{A}=1$-nonanol, and $\mathrm{B}=1$-octanol), despite symmetric highest generalization between 1-octanol and 1-nonanol (see Fig. 3). Similarly, the results of the generalization matrix predicted highest asymmetric generalization from 1-nonanol to limonene. In none of the two odor combinations involving $\mathrm{A}=1$-nonanol and $\mathrm{B}=$ limonene blocking was found.

Augmentation-like test performances $\left(\boldsymbol{B}_{\text {block }}>B_{\text {novel }}\right.$ )

By using a blocking design, Batsell et al. $(1999,2001)$ have reported that an effect opposite to blocking may also occur, i.e., augmented conditioning to $\mathrm{B}$ in the $\mathrm{A}+\mathrm{AB}+$ design. They have called this effect "augmentation." In our experiments, we found two odor combinations out of the 24 studied consistent with the definition of augmentation because the response in the test was significantly higher in the block than in the novel group (Table

\section{Learning \& Memory}

www.learnmem.org 
Table 2. The 24 odor combinations used in experiment 1

\begin{tabular}{|c|c|c|}
\hline Odor combination & $\begin{array}{c}\text { Test } \\
\text { performance }\end{array}$ & $\begin{array}{c}\text { Sixth } A+ \\
\text { vs. } \\
\text { first } A B+\end{array}$ \\
\hline 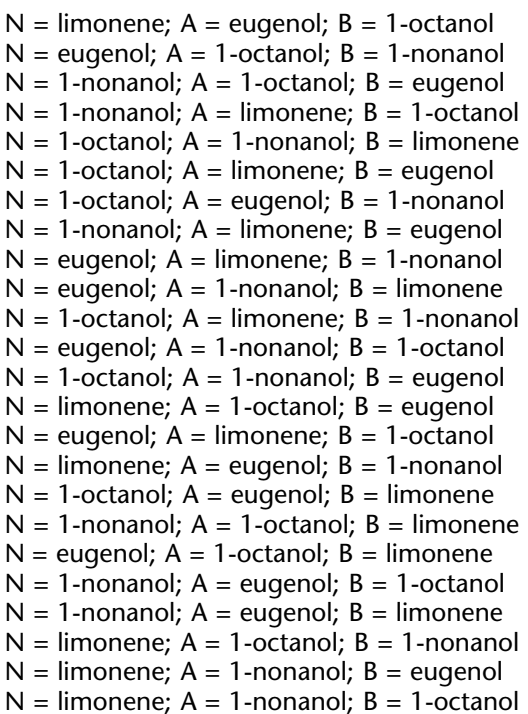 & $\begin{array}{l}\text { blocking } \\
\text { blocking } \\
\text { blocking } \\
\text { blocking } \\
\text { augmentation } \\
\text { augmentation } \\
\text { NS } \\
\text { NS } \\
\text { NS } \\
\text { NS } \\
\text { NS } \\
\text { NS } \\
\text { NS } \\
\text { NS } \\
\text { NS } \\
\text { NS } \\
\text { NS } \\
\text { NS } \\
\text { NS } \\
\text { NS } \\
\text { NS } \\
\text { NS } \\
\text { NS } \\
\text { NS }\end{array}$ & $\begin{array}{l}N S \\
N S \\
N S \\
A>A B \\
A>A B \\
A>A B \\
A>A B \\
A>A B \\
A>A B \\
A>A B \\
A>A B \\
A>A B \\
A>A B \\
A>A B \\
A>A B \\
A>A B \\
N S \\
N S \\
N S \\
N S \\
N S \\
N S \\
N S \\
N S\end{array}$ \\
\hline
\end{tabular}

Test performance (midcolumn) indicates blocking when the response to $B$ in the test was significantly higher in the novel than in the block group. It indicates augmentation when the response to $B$ in the test was significantly higher in the block than in the novel group. NS indicates no significant differences. The right column shows the cases in which the response in the last trial of the first preconditioning phase was significantly higher than that in the first trial of the compound phase. In all the cases $\mathrm{N}=20$ per group, and $\alpha$ was set at 0.05 .

2; mid column; $P<0.05$ in both cases). These were as follows: (1), $\mathrm{N}=1$-octanol, $\mathrm{A}=1$-nonanol, and $\mathrm{B}=$ limonene, and (2) $\mathrm{N}=1$ octanol, $\mathrm{A}=$ limonene and $\mathrm{B}=$ eugenol. In both odor combinations, the response level in the last compound-conditioning trial $(\mathrm{AB}+)$ was significantly higher in the block than in the novel group. Thus, the two cases of augmentation may simply reflect the fact that at the end of the compound-conditioning phase, the response level of the block group was higher than that of the novel group. Accordingly, a higher level of responses to B can be expected in the block than in the novel group in the test phase, and this is exactly what we found. Again, the argument of similarity between A and B cannot account for this effect. Although generalization from 1-nonanol to limonene was highest (see Fig. $3)$, it was smallest between limonene and eugenol.

\section{Nonsignificant test performances $\left(B_{\text {block }}=B_{\text {novel }}\right)$}

In the majority of the cases (18 out of 24 odor combinations), we found no significant difference between the novel and the block groups in the test phase $(P<0.05$; mid column; Table 2$)$. From these 18 cases, two deserve a special mention. In the odor combinations $\mathrm{N}=1$-octanol, $\mathrm{A}=$ eugenol, and $\mathrm{B}=$ limonene; and $\mathrm{N}=1$-octanol $\mathrm{A}=$ eugenol, and $\mathrm{B}=1$-nonanol, there were no differences between the novel and the block groups in the test phase, but the level of responses for both groups was remarkably low $(<50 \%)$. Acquisition for $\mathrm{AB}+$ in the compound-conditioning phase was poor both for the novel and the block groups in both odor combinations. This result is interesting because there was no blocking $\left(\mathrm{B}_{\text {block }}=\mathrm{B}_{\text {novel }}\right.$ in the test $)$. This, however, might be because learning about $\mathrm{B}$ was equally deficient in both cases. Therefore, it seems inappropriate to conclude that learning about B was not impaired in the block group (i.e., absence of blocking) as it was in fact impaired in both the novel and the block group. By using a different behavioral response (duration of PER), Hosler and Smith (2000) concluded absence of blocking in two cases in which the test responses of the block and the novel group were similar but extremely low (even lower than stimulus duration; see their Fig. 4B,C). As explained above, this conclusion seems inappropriate.

\section{Experiment 2}

Experiment 1 established that odor similarity is not determinant for blocking in the honeybee. As a different conclusion was reached by Hosler and Smith (2000) (see Introduction), we intended to replicate their results by using the same the three odors as in their work: 1-hexanol, 1-octanol, and geraniol (Fig. 1C). We presented these odors in the same six combinations used by Hosler and Smith (2000; Table 3). To increase the statistical robustness of our analyses, and to compensate for any presumed lower sensitivity of the \% PER measure compared with the response duration measure, we included 20 individuals per group (and not 10).

\section{Blocking: Performance and odor combination}

We focused directly on each odor combination to analyze the effect of odor similarity on blocking. Contrarily to Hosler and Smith (2000), we did not find any blocking effect (Table 3). This is valid both for comparisons using an $\alpha$ value of 0.05 and a corrected $\alpha$ level of $0.0083(0.05 / 6)$. In five out of six odor combinations, there were no significant differences in the response to $B$ in the test between the block and novel groups. In the remaining case (N, 1-octanol; A, geraniol; $\mathrm{B}, 1$-hexanol), we found a significant augmentation effect if an $\alpha$ of 0.05 is taken $\left(F_{(1,38)}=5.18, P<0.03\right)$, but no effect if the corrected $\alpha$ of 0.0083 is used. In either case, the result is different from that obtained by Hosler and Smith (2000) for the same odor combination. These investigators reported that blocking occurs as follows: N, 1-octanol; A, geraniol; and B, 1-hexanol (see first panel top left in their Fig. 4). It is important to mention that the behavioral variables in their and our experiments are different: we used the \% PER while they used the mean duration of PER, which is presumably more sensitive to detect differences in performance. However, also the $\%$ PER measure yields a result, but one opposite to that reported by Hosler and Smith (2000) in the case of PER duration; thus, the argument on reduced sensitivity of the variable used to measure the bees' response does not apply to our analyses.

In four out of six combinations $(\mathrm{N}=1$-octanol, $\mathrm{A}=$ geraniol, $\mathrm{B}=1$-hexanol; $\mathrm{N}=1$-hexanol, $\mathrm{A}=$ geraniol, $\mathrm{B}=1$-octanol; $\mathrm{N}=$ geraniol, $\mathrm{A}=1$-hexanol, $\mathrm{B}=1$-octanol; and $\mathrm{N}=$ geraniol, $\mathrm{A}=1$-octanol, $\mathrm{B}=1$-hexanol), the response in the first trial of the compound-conditioning phase was significantly lower than in the last preconditioning phase ( $P<0.05$, in all cases). If an $\alpha$ of 0.0083 is used, differences remain significant only in the follow-

Table 3. The six odor combinations used in experiment 2

\begin{tabular}{lcc}
\hline Odor combination & $\begin{array}{c}\text { Test } \\
\text { performance }\end{array}$ & $\begin{array}{c}\text { Sixth } A+ \\
\text { vs. } \\
\text { first } A B+\end{array}$ \\
\hline$N=1$ octanol; $A=$ geraniol; $B=1$-hexanol & augmentation & $\mathrm{A}>\mathrm{AB}$ \\
$\mathrm{N}=1$-octanol; $\mathrm{A}=1$-hexanol; $\mathrm{B}=$ geraniol & $\mathrm{NS}$ & $\mathrm{NS}$ \\
$\mathrm{N}=1$-hexanol; $\mathrm{A}=1$-octanol; $\mathrm{B}=$ geraniol & $\mathrm{NS}$ & $\mathrm{NS}$ \\
$\mathrm{N}=1$-hexanol; $\mathrm{A}=$ geraniol; $\mathrm{B}=1$-octanol & $\mathrm{NS}$ & $\mathrm{A}>\mathrm{AB}$ \\
$\mathrm{N}=$ geraniol; $\mathrm{A}=1$-hexanol; $\mathrm{B}=1$-octanol & $\mathrm{NS}$ & $\mathrm{A}>\mathrm{AB}$ \\
$\mathrm{N}=$ geraniol; $\mathrm{A}=1$-octanol; $\mathrm{B}=1$-hexanol & $\mathrm{NS}$ & $\mathrm{A}>\mathrm{AB}$ \\
\hline
\end{tabular}

Same conventions as in Table 2. In all the cases $\mathrm{N}=20$ per group and $\alpha$ was set at 0.05 . 
ing case: N, 1-octanol; A, geraniol; B, 1-hexanol $\left(F_{(1,19)}=10.23\right.$; $P<0.005)$.

\section{Discussion}

Our results show that blocking is not a robust phenomenon in olfactory conditioning of the PER in honeybees and, more importantly, that similarity between odors A and B is not determinant for the occurrence of blocking. The first conclusion is supported by the fact that a large number of bees (960) was necessary in experiment 1 to detect the blocking effect in the pooled performances of the block and the novel groups (response to $\mathrm{B}$ in the test phase; see Fig. 4, test). Also, an attempt to directly replicate the results from Hosler and Smith (2000) failed, supporting the conclusion that the occurrence of blocking in honeybee olfactory learning is not a robust phenomenon. The second conclusion was reached after studying the performance of the block and the novel groups in each odor combination used in experiment 1 . We applied a noncorrected $\alpha$ in order to facilitate comparison with the work of Hosler and Smith (2000) and found that odor similarity did not determine the occurrence of blocking. Note that blocking would not be found in any of the 24 odor combinations tested in experiment 1 if a corrected $\alpha$ were applied. In experiment 2, blocking was not found in any of the six odor combinations tested. These results underline the inconsistency of the blocking phenomenon in honeybee olfactory learning. Hosler and Smith (2000) postulated that perceptual similarity between the odors $\mathrm{A}$ and $\mathrm{B}$ is the critical feature determining blocking. Following the method of Linster and Smith (1997), they argued that if A and B are perceptually similar, first-phase training with A engages a neural pathway for which $B$ has later on to compete due to its similarity to A. In this case, neural coding of B will be impaired thus leading to a blocking effect. Our results show that this conclusion is not tenable.

Being nonconservative in the choice of the $\alpha$ level $(\alpha=0.05$ ), we found evidence for blocking in four out of 24 odor combinations of experiment 1 . These observed differences in responding to B between groups, however, were not related to the similarity between A and B. Neither the trend analysis (Fig. 5) nor the conventional blocking analyses at the level of individual odor combinations supported the similarity argument. Compared with the method of Hosler and Smith (2000) we used four instead of three odors and we assessed the similarity of the four odors in independent groups. This enhanced the number of odor combinations under study from six (Hosler and Smith 2000) to 24 (this study). Furthermore, our database for the evaluation of the effect of similarity on blocking consisted of 960 bees compared with 60 bees in the case of Hosler and Smith (2000). Hence, the power for detecting a potential influence of the degree of similarity between the elements of an olfactory compound on blocking was high. Nevertheless, we could not find reliable hints for this postulated impact. The same conclusion applies to experiment 2, which constituted a replication of Hosler and Smith's (2000) work. Despite the fact that the odor combinations were identical in both studies, we obtained results that were different from those of Hosler and Smith (2000). This difference cannot be attributed to the fact that different variables were used to measure the response of the bees in both works. Moreover, we performed experiment 2 in such a way that our database was twice as large as that of Hosler and Smith (2000). Thus, the power for detecting a potential influence of the degree of similarity between the odors of an olfactory compound on blocking was also high in experiment 2 . We thus conclude that similarity between $\mathrm{A}$ and $\mathrm{B}$ is not a determinant factor for blocking in the olfactory domain, and that blocking itself is not a widespread and reliable phenomenon in olfactory PER conditioning of bees. Given this conclusion, it seems important that further studies on blocking, and more generally on olfactory conditioning of PER, involving multiple groups include corrected $\alpha$ levels in order to ensure correct interpretations of the experimental data.

The conclusion about the lack of reliability of the blocking phenomenon is in line with that of Gerber and Ullrich (1999), who could also not find convincing evidence for blocking in the same experimental context. The conclusions of Gerber and Ullrich (1999) were raised on the basis of a general analysis, which, except for the case of geraniol and 1-hexanol, did not systematically take into account the specific case of each odor combination tested. In their case, no blocking was found (i.e., no experiment yielded a response to B in the block group smaller than that of the novel group), when confounding factors were eliminated.

When analyzing performance in a blocking design, it would be also important to consider the potential effects introduced by the novel odorant $(\mathrm{N})$ design. If there is any generalization from the novel odor to the blocking (A) or blocked (B) odorant, or if there is generalization from $A$ to $B$, this could attenuate the ability to detect blocking. If $A$ and $B$ are going to be similar or dissimilar as a treatment effect, at least $\mathrm{N}$ must be dissimilar to both. Detection of blocking can thus depend on $\mathrm{N}$ as much as it depends on A and B.

Hosler and Smith's (2000) similarity argument was originally postulated by Linster and Smith (1997), who proposed a model based on neural connectivity in the antennal lobe, the primary olfactory neuropile in the insect brain. Within this structure, sensory cells synapse onto projection neurons, which then carry information out of the first layer for processing in higherorder neuropiles such as the lateral protocerebrum and the mushroom bodies. Each antennal lobe is constituted by 160 glomeruli, the glomeruli being convergence centers between sensory and projection neurons and local interneurons. Local interneurons potentially shape the information carried by the projection neurons. These interneurons, which can contact all glomeruli (homogeneous local interneurons) or only some of them (heterogeneous local interneurons) (Fonta et al. 1993), are GABAergic and exert inhibitory effects on glomerular activity. The model by Linster and Smith (1997) incorporated these different cell types and a biologically inspired modulatory neuron with which modifiable Hebb-like synaptic interactions take place. A learning rule that incorporated modifiable connections from projection neurons onto the modulatory neuron was sufficient to account for behavioral olfactory generalization and overshadowing in bees (Smith 1997b). A second type of excitatory connection from the modulatory neuron onto local inhibitory interneurons was necessary to reproduce behavioral results from blocking, under the assumption that similar odors result in blocking-like performances (Linster and Smith 1997; Hosler and Smith 2000). As our results demonstrate, this assumption was wrong and the model needs to be revised. In fact, Linster and coworkers have recently initiated this revision studying olfactory blocking in rats (Giannaris et al. 2002). Their experimental work did not yield the results predicted by the model of Linster and Smith (1997) as blocking occurred independently of odor similarity, albeit to a higher magnitude when odors were more dissimilar (Wiltrout et al. 2003).

The analysis of the transition from the end of the first training phase (preconditioning with A) to the beginning of the second training phase (compound conditioning with $\mathrm{AB}$ ) in the block groups yielded a significant effect that deserves further comment. The level of responding in the first $\mathrm{AB}$ trial compared to that in the last A trial was clearly influenced by odor combination. This observation contradicts a mere elemental summation principle such as the Rescorla-Wagner theory (1972), which assumes that a compound $\mathrm{AB}$ is processed as the mere sum of its 
separate components such that the associative strength $(\mathrm{V})$ of the compound equals the sum of the associative strengths of its components $\left(\mathrm{V}_{\mathrm{AB}}=\mathrm{V}_{\mathrm{A}}+\mathrm{V}_{\mathrm{B}}\right)$. If this would be true, bees should respond to $\mathrm{AB}$ in the same manner as to $\mathrm{A}$ regardless of odor combination, because the unknown stimulus $B$ should have no associative strength and only A would contribute to the associative strength of the compound. Configural theories (see Pearce 1987, 1994), on the other hand, which propose that a compound constitutes an entity different from its components $(\mathrm{AB}=\mathrm{X} \neq \mathrm{A}+\mathrm{B})$, are more likely to predict the outcome of our experiments. Pearce's configural theory would predict generalization from $\mathrm{A}$ to $\mathrm{AB}$ depending on their similarity. Similarity sensu Pearce is defined in terms of the number of common elements. Thus, Pearce's configural theory predicts that responding to $\mathrm{AB}$ should be lower than responding to $\mathrm{A}$, but, without further assumptions, would not predict that this decrement in responding is modulated by odor combination. In our data, however, the effect of odor combination was much stronger than the effect of a general decrement in responding. Finally, a third view is proposed by the unique-cue theory, which states that a compound is processed as the sum of its components plus a stimulus $(u)$ that is unique to the joint presentation of the elements in the mixture $(\mathrm{AB}=\mathrm{A}+\mathrm{B}+\mathrm{u})$ (Whitlow and Wagner 1972). Deisig et al. (2001, 2002,2003 ) have shown that a modified unique-cue theory accounts for olfactory compound processing and learning in honeybees. This theory has the same assumptions as the unique-cue theory but assumes additionally that components presented in a compound are less salient than the presentation of the same elements separately because elements within a compound might interfere with each other (Mackintosh 1971; James and Wagner 1980). Such an interference may underlie the decrement in response observed in the transition from the end of the first training phase (preconditioning with A) to the beginning of the second training phase (compound conditioning with $\mathrm{AB}$ ) in the block groups.

We have proposed (Deisig et al. 2002; Giurfa 2003; Lachnit et al. 2004) that the neural correlate of a unique cue in olfactory compound processing by honeybees is the specific odor suppression phenomenon that can be recorded at the level of the antennal lobe when the bee is stimulated with an odor mixture. By using optical imaging techniques in which $\mathrm{Ca}^{2+}$-sensitive dyes are infused into the antennal lobe of the honeybee has allowed, uncovering the principles of olfactory coding in bees (Joerges et al. 1997) and visualizing the suppression phenomenon in the case of olfactory mixtures. It was shown that each odor is encoded as a specific spatial glomerular activation pattern (Joerges et al. 1997; Galizia and Menzel 2000). Interestingly, activity patterns induced by olfactory compounds deviate more or less from the patterns that would be expected if simple linear superposition governed neural processing. Both the kind of odors and the number of odors mixed together appear to play a role (Joerges et al. 1997). However, these results were obtained by means of bath application of calcium green (Joerges et al. 1997). This technique records the combined activity of several neuronal populations of the antennal lobe, among which primary-afferent activity seems to have an important contribution. As the antennal lobe circuitry transforms the primary-afferent representations of odors (Sachse and Galizia 2003), recordings where primary-afferent receptor activity is predominant are not necessarily useful to evaluate olfactory compound processing. New methods have been developed, which allow to record selectively the calcium activity of the efferent projection neurons by means of selective staining with fura-dextran, a ratiometric dye (Sachse and Galizia 2002, 2003). Thus, studies applying this technique are necessary to study how olfactory compounds varying in the number of components are processed. The existence of odor-specific suppression phenomena due to nonlinear processing in the antennal lobe network could then be studied.

Applying an integrative view that combines the knowledge derived from neurobiological studies on honeybee olfaction and that from psychological studies on honeybee learning will help to uncover the regularities, if any, underlying the impact of odor combinations observed. How the conditioning phases implied in a blocking experiment modify the elemental and compound olfactory representations at the level of the antennal lobe remains to be studied. In any case, future work can take advantage of these optophysiological techniques to relate behavioral performance to neural activity in the olfactory network (Guerrieri et al. 2005).

We thus conclude that a complete behavioral analysis, including not only the performance in the test phase but also those in the preceding acquisition phases, is mandatory to produce a comprehensive study of blocking. Such a study should lead to specific hypotheses about stimulus processing and representation that could be tested by using physiological approaches: In the case of olfactory conditioning of PER in honeybees, we have underlined the pertinence of imaging techniques, as a possible tool to test the validity of such hypotheses. Such a combined approach will yield useful information about the mechanisms underlying blocking-like or augmentation-like performances, provided that robust, reproducible effects of blocking or augmentation can indeed be observed. In this respect, it is worth citing the words of Rescorla and Holland (1982), who enormously contributed to the study of blocking from a psychological perspective and who concluded that "one begins to fear that additional cases that would be important to our understanding about conditioning could be ignored because the blocking phenomenon, rather than the processes which it suggests, has gained dominance in our thinking."

\section{Materials and Methods}

\section{Subjects}

Free-flying honeybee foragers, Apis mellifera, were caught when leaving the hive in the morning of every experimental day. They were placed in small glass vials and cooled down on ice until they stopped moving. The bees were then harnessed in small metal tubes such that they could only move their antennae and mouthparts, including the proboscis. Bees were kept in the dark and in high humidity for approximately $3 \mathrm{~h}$. Before starting the experiments, each subject was checked for intact PER by lightly touching the antennae with a toothpick imbibed with sucrose solution without subsequent feeding. Extension of the proboscis beyond a virtual line between the open mandibles was counted as PER (unconditioned response). Animals that did not show the reflex were discarded.

\section{US and CS}

The US was a $50 \%(\mathrm{w} / \mathrm{w})$ sucrose solution. In experiment 1 , the odors chosen as CSs were 1-octanol, 1-nonanol, eugenol, and limonene (Fig. 1A). They were chosen on the basis of their chemical structures (1-octanol and 1-nonanol are structurally similar, while eugenol and limonene are not and also differ from 1-octanol and 1-nonanol) and because their physiological coding properties at the level of the bee antennal lobe is known (Sachse et al. 1999). These odors evoke different glomerular activation patterns of varying similarity (Fig. 1B). These patterns suggest that 1-octanol and 1-nonanol should be perceptually similar for the bees. Eugenol and limonene should differ perceptually from each other and from 1-octanol and 1-nonanol. Such predictions were explicitly tested by means of a behavioral generalization matrix (see below). In experiment 2, the odors chosen as CSs were the same used by Hosler and Smith (2000): 1-hexanol, 1-octanol, and geraniol (Fig. 1C). 
Five microliters of pure odor were applied onto a fresh strip of filter paper $\left(1 \mathrm{~cm}^{2}\right)$. Each paper strip was imbibed before the beginning of each training phase and then placed into a $20-\mathrm{mL}$ plastic syringe. For the cases in which a compound of two odors was used as CS, $5 \mu \mathrm{L}$ of each odor were applied on the same filter paper strip.

\section{Procedure}

Each trial lasted $60 \mathrm{sec}$. At the beginning of each trial the subject harnessed in its individual tube was placed in the experimental holder, between an air extractor and the syringe for $25 \mathrm{sec}$ to allow familiarization with the training situation. The air extractor behind the bee impeded the accumulation of residual odors. After the 25 initial seconds, the CS was released for $5 \mathrm{sec}$. In reinforced trials, the US onset occurred $3 \mathrm{sec}$ after CS onset and lasted for $5 \mathrm{sec}$. The interstimulus interval was $3 \mathrm{sec}$, and the overlap between CS and US was 2 sec. To release the US, both antennae were lightly touched with a toothpick imbibed in the sucrose solution, and the bee was allowed to lick the toothpick after proboscis extension. This was constant for all bees because even if the bees extended the proboscis to the odor itself, they got nevertheless the antennal stimulation with the toothpick. The bee was then left in the holder until completing $60 \mathrm{sec}$. It was thereafter returned to its resting position. The intertrial interval was $10 \mathrm{~min}$.

We recorded whether or not a bee extended its proboscis after onset of the odor (CS) and before presentation of the sucrose solution (US). Multiple responses during a CS were counted as a single PER. We also recorded the response to the US at the end of a conditioning phase to check for intact PER. If a bee did not respond to the US, it was discarded.

\section{Experiment 1: Behavioral generalization matrix}

Each odor used in experiment 1 was paired with sucrose in the course of six conditioning trials. Ten minutes after the last conditioning trial, bees were presented with a different odorant in a single extinction test (no US delivery). A different group of bees was used for each possible odor combination. Twenty bees were trained and tested per odor pair. Twelve groups of 20 bees each were used ( 240 bees).

\section{Experiments 1 and 2: Blocking}

The design of the blocking experiments followed that of Hosler and Smith (2000) and was the same both in experiments 1 and 2. For each odor set, we studied two groups during two conditioning phases (Table 1). First, in the first training phase (preconditioning) phase, each group was conditioned with a single odorant in the course of six forward CS-US pairing trials. One group (block) was preconditioned with odorant A, while the second group (novel) was preconditioned with a different odorant $\mathrm{N}$. During the second training phase, the compound-conditioning phase, both groups were conditioned with a compound made of the two odorants $\mathrm{AB}$ along six forward CS-US pairing trials. Ten minutes after the last conditioning trial, both groups were tested with B in a single extinction trial (no US delivery). The response to B in both groups was then compared. If blocking occurs, the response to $\mathrm{B}$ should be lower in the block group than in the novel group. Both groups differed with respect to the role of $A$ as predictor of reward at the onset of the compound-conditioning phase. Subjects in both groups experienced B in an identical manner. Odors were used equally often and were counterbalanced across days, as N, A, and B. In experiment 1, 24 independent experiments (i.e., 24 block and 24 novel groups) were performed (see Table 2); in experiment 2, six independent experiments (i.e., six block and six novel groups) were performed (see Table 3).

\section{Statistical analysis}

For the analyses of the behavioral matrix (experiment 1), we first tested whether the response to test odors was homogeneous. We used a $\chi^{2}$ analysis to compare the responses to the rewarded odor- ant to those to the other odors (null hypothesis: response frequency $1 / 4: 3 / 4) . \chi^{2}$ analysis was also used to test the symmetry of generalization between odors, i.e., to compare the response to $\mathrm{B}$ after training of $\mathrm{A}$ and the response to $\mathrm{A}$ after training to $\mathrm{B}$. Finally we ranked generalization performance between odors by subdividing the $\chi^{2}$ analysis.

For the analyses of blocking both in experiments 1 and 2, parametric analyses of variance (ANOVA) were used for comparisons and trend tests. Although parametric ANOVA is not allowed in case of dichotomous data such as those of the PER, Monte Carlo studies have shown that it is permissible to use ANOVA under certain conditions (the proportion of responses in the smaller response category is $\geq 0.2$ and there are at least 20 degrees of freedom for error) (Lunney 1970), which were met in our experiments. In addition, we computed power analyses (partial $\eta^{2}$ ) (see Cohen 1988) in order to evaluate the size of the effects observed. Two $\alpha$ levels were employed for the individual odor combinations of experiments 1 and 2: an $\alpha$ of 0.05 , which was the one used by Hosler and Smith (2000), and a corrected one (0.0021 in experiment 1 and 0.0083 in experiment 2 ) for the case of multiple comparisons ( 24 in experiment 1 ; six in experiment 2 ).

\section{Acknowledgments}

We thank two anonymous referees and Jean-Marc Devaud, Giovanni Galizia, Christiane Linster, Jean-Christophe Sandoz, Brian Smith, and Marco Schubert for useful comments and correction on previous versions of the manuscript. Thanks are due also to Vanessa Bordier, Thierry Nohasiarivelo, Bernhard Komischke, Gladys Krause, Geoffrey Portelli, Dagmar Malun, and France Rousseau for help and support, and to Cecilia Mendive for providing Figs. 1A and 1C. F.G. was partially supported by the José Estenssoro Program of the Fundación YPF (Argentina). Support to B.G. comes from the Deutsche Forschungsgemeinschaft, SFB 554 This work was supported by funds of the Human Frontier Science Program, the Fondation pour la Recherche Médicale, and a preproject of the ACI Integrative and Computational Neurosciences (French Research Ministry).

\section{References}

Allen, M.T., Padilla, Y., and Gluck, M.A. 2002. Blocking in rabbit eyeblink conditioning is not due to learned inattention: Indirect support for an error correction mechanism of blocking. Integr. Physiol. Behav. Sci. 37: 254-264.

Arcediano, F., Matute, H., and Miller, R.R. 1997. Blocking of pavlovian conditioning in humans. Learn. Motiv. 28: 188-199.

Batsell, W.R. 1996. Retention of context blocking in taste-aversion learning. Physiol. Behav. 61: 437-446.

Batsell, W.R. and Batson, J. 1999. Augmentation of taste conditioning by a preconditioned odor. J. Exp. Psychol. Anim. Behav. Proc. 25: $374-388$.

Batsell, W.R., Paschall, G.Y., Gleason, D.I., and Batson, J.D. 2001. Taste preconditioning augments odor-aversion learning. J. Exp. Psychol. Anim. Behav. Proc. 27: 30-47.

Beauchamp, A.J., Gluck, J.P., Fouty, H.E., and Lewis, M.H. 1991. Associative processes in differentially reared rhesus monkeys (Macao mulatta): Blocking. Dev. Psychobiol. 24: 175-189.

Bitterman, M., Menzel, R., Fietz, A., and Schäfer, S. 1983. Classical conditioning of proboscis extension in honeybees (Apis mellifera). J. Comp. Psychol. 97: 107-119.

Brembs, B. and Heisenberg, M. 2001. Conditioning with compound stimuli in Drosophila melanogaster in the flight simulator. J. Comp. Biol. 204: 2849-2859.

Cohen, J. 1988. Statistical power analysis for the behavioral sciences, 2nd ed. Lawrence Erlbaum Associates, Hillsdale, NJ.

Couvillon, P.A., Arakaki, L., and Bitterman, M.E. 1997. Intramodal blocking in honeybees. Anim. Learn. Behav. 25: 277-282.

Couvillon, P.A., Campos, A.C., Bass, T.D., and Bitterman, M.E. 2001. Intermodal blocking in honeybees. Q. J. Exp. Psychol. 54B: 369-381.

Deisig, N., Lachnit, H., Giurfa, M., and Hellstern, F. 2001. Configural olfactory learning in honeybees: Negative and positive patterning discrimination. Learn. Mem. 8: 70-78.

Deisig, N., Lachnit, H., and Giurfa, M. 2002. The effect of similarity between elemental stimuli and compounds in olfactory patterning discriminations. Learn. Mem. 9: 112-121. 
Deisig, N., Lachnit, H., Sandoz, J.C., Lober, K., and Giurfa, M. 2003. A modified version of the unique cue theory accounts for olfactory compound processing in honeybees. Learn. Mem. 10: 199-208.

Fonta, C., Sun, X., and Masson, C. 1993. Morphology and spatial distribution of bee antennal lobe interneurones responsive to odours. Chem. Senses 18: 101-119.

Funayama, E.S., Couvillon, P.A., and Bitterman, M.E. 1995. Compound conditioning in honeybees: Blocking tests of the independence assumption. Anim. Learn. Behav. 23: 429-437.

Galizia, C.G. and Menzel, R. 2000. Odour perception in honeybees: Coding information in glomerular patterns. Curr. Opin. Neurobiol. 10: $504-510$.

Gerber, B. and Ullrich, J. 1999. No evidence for olfactory blocking in honeybee classical conditioning. J. Exp. Biol. 202: 1839-1854.

Giannaris, E.L., Cleland, T., and Linster, C. 2002. Intramodal blocking between olfactory stimuli in rats. Physiol. Behav. 75: 717-722.

Giftakis, J.E. and Tait, R.W. 1998. Blocking of the rabbit's nictitating membrane response: Effects of modifications of contextual associative strength. Learn. Motiv. 29: 23-48.

Giurfa, M. 2003. Cognitive neuroethology: Dissecting non-elemental learning in a honeybee brain. Curr. Opin. Neurobiol 13: 726-735.

Good, M. and Mcphail, E.M. 1994. Hippocampal lesions in pigeons (Columba livia) disrupt reinforced preexposure but not overshadowing or blocking. Q. J. Exp. Psychol. 47: 263-291.

Guerrieri, F., Schubert, M., Sandoz, J.-C., and Giurfa, M. 2005. Perceptual and neural olfactory similarity in honeybees. PLoS Biol. 3: $\mathrm{e} 60$.

Hosler, J. and Smith, B. 2000. Blocking and the detection of odor components in blends. J. Exp. Biol. 203: 2797-2806.

James, J.H. and Wagner, A.R. 1980. One trial overshadowing evidence of distributive processing. J. Exp. Psychol. Anim. Behav. Process. 6: $188-205$.

Joerges, J., Küttner, A., Galizia, C.G., and Menzel, R. 1997. Representations of odours and odour mixtures visualized in the honeybee brain. Nature 387: 285-287.

Kamin, L.J. 1968. In Miami Symposium on the prediction of behavior: Aversive stimulation (ed. M.R. Jones), pp. 9-31. University of Miami Press, Miami, FL.

1969. Predictability, surprise, attention and conditioning. In Punishment and aversive behavior (eds. B. Campbell and R. Church), pp. 279-298. Appleton-Century-Crofts, New York.

Lachnit, H., Giurfa, M., and Menzel, R. 2004. Odor processing in honey bees: Is the whole equal to, more than, or different from the sum of its parts? In Advances in the study of behavior, Vol. 34 (eds. P.J.B. Slater et al.), pp. 241-295. Elsevier Academic Press, San Diego, CA.

Linster, C. and Smith, B. 1997. A computational model of honey bee antennal lobe circuitry to odor mixtures: Overshadowing, blocking and unblocking can arise from lateral inhibition. Behav. Brain Res. 87: $1-14$.

Lunney, G.H. 1970. Using analysis of variance with a dichotomous dependent variable: An empirical study. J. Educ. Meas. 7: 263-269.

Mackintosh, N.J. 1971. Reward and aftereffects of reward in the learning of goldfish. J. Comp. Physiol. Psychol. 76: 225-232.

. 1975. The psychology of animal learning. Academic Press, New York.

Miller, R.R. and Matute, H. 1996. Biological significance in forward and backward blocking: Resolution of discrepancy between animal conditioning and human casual judgment. J. Exp. Psychol. 125: $370-386$.

Miller, R.R. and Matzel, L.D. 1988. The comparator hypothesis: A response rule for the expression of associations. In The psychology of learning and motivation, vol. 22 (ed. G.H. Bower), pp. 51-92. Academic Press San Diego, CA.
Pavlov, I. 1927. Conditioned reflexes. Oxford University Press, Oxford, UK.

Pearce, J.M. 1987. A model of stimulus generalization in Pavlovian conditioning. Psychol. Rev. 94: 61-73.

. 1994. Similarity and discrimination: A selective review and a connectionist model. Psychol. Rev. 101: 587-607.

Pearce, J.C. and Hall, G. 1980. A model for pavlovian learning: Variations in the effectiveness of conditioned but not of unconditioned stimuli. Psychol. Rev. 87: 532-552.

Rescorla, R.A. and Holland, P.C. 1982. Behavioral studies of associative learning in animals. Ann. Rev. Psychol. 33: 265-308.

Rescorla, R.A. and Wagner, A.R. 1972. A theory of pavlovian conditioning: Variations in the effectiveness of reinforcement and nonreinforcement. In Classical conditioning II (eds. A. Black and W.R. Prokasy), pp. 64-99. Academic Press, New York.

Rogers, R.F. and Matzel, L.D. 1996. Higher-order associative processing in Hermissenda suggests multiple sites of neuronal modulation. Learn. Mem. 2: 279-298.

Sachse, S. and Galizia, C.G. 2002. Role of inhibition for temporal and spatial odor representation in olfactory output neurons: A calcium imaging study. J. Neurophysiol. 87: 1106-1117.

. 2003. The coding of odour-intensity in the honeybee antennal lobe: Local computation optimizes odour representation. Eur. J. Neurosci. 18: 2119-2132.

Sachse, S., Rappert, A., and Galizia, C.G. 1999. The spatial representation of chemical structures in the antennal lobe of the honeybees: Steps toward the olfactory code. Eur. J. Neurosci. 11: $3970-3982$.

Sahley, C., Rudy, J.W., and Gelperin, A. 1981. An analysis of associative learning in a terrestrial mollusc: Higher-order conditioning, blocking and a transient US pre-exposure effect. J. Comp. Physiol. 144: 1-8.

Smith, B. 1997a. An analysis of blocking in binary odorant mixtures: An increase but not a decrease in intensity of reinforcement produces unblocking. Behav. Neurosci. 111: 57-69.

. 1997b. Analysis of interaction in binary odorant mixtures. Physiol. Behav. 65: 397-407.

Smith, B. and Cobey, S. 1994. The olfactory memory of the honey bee Apis mellifera, II: Blocking between odorants in binary mixtures. J. Exp. Biol. 195: 91-108.

Solomon, P.R. 1977. Role of the hippocampus in blocking and conditioned inhibition of the rabbit's nictitating membrane response. J. Comp. Psychol. 91: 407-417.

Sutherland, N.S. and Mackintosh, N.J. 1971. Mechanisms of animal discrimination learning. Academic Press, New York.

Takeda, K. 1961. Classical conditions response in the honey bee. J. Ins. Physiol. 6: 168-179.

Thorn, R. and Smith, B. 1997. The olfactory memory of the honey bee Apis mellifera, III: Bilateral sensory input is necessary for induction and expression of olfactory blocking. J. Exp. Biol. 200: 2045-2055.

Von Frisch, K. 1967. The dance language and orientation of bees. Harvard University Press, Cambridge, MA.

Waelti, P., Dickinson, A., and Schultz, W. 2001. Dopamine responses comply with basic assumptions of formal learning theory. Nature 412: $43-48$.

Wiltrout, C., Dogra, S., and Linster, C. 2003. Configurational and nonconfigurational interactions between odorants in binary mixtures. Behav. Neurosci. 117: 236-245.

Whitlow, J.W. and Wagner, A.R. 1972. Negative patterning in classical conditioning: Summation of response tendencies to isolate and configural components. Psychol. Sci. 27: 299-301.

Received April 5, 2004; accepted in revised form February 8, 2005. 


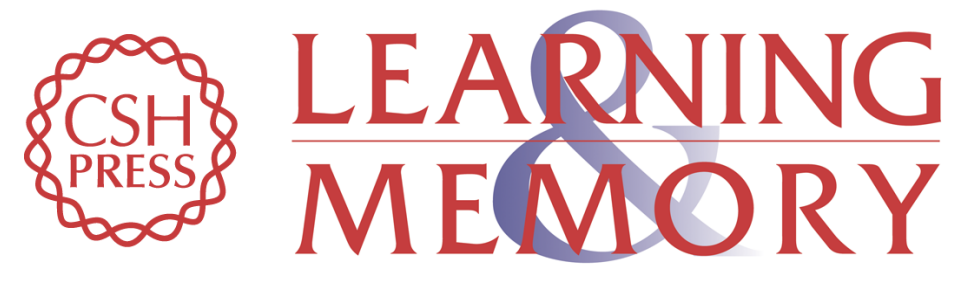

\section{Olfactory blocking and odorant similarity in the honeybee}

Fernando Guerrieri, Harald Lachnit, Bertram Gerber, et al.

Learn. Mem. 2005, 12:

Access the most recent version at doi:10.1101/lm.79305

References This article cites 42 articles, 8 of which can be accessed free at: http://learnmem.cshlp.org/content/12/2/86.full.html\#ref-list-1

License

Email Alerting Receive free email alerts when new articles cite this article - sign up in the box at the Service top right corner of the article or click here. 